$>$ L'accident vasculaire cérébral (AVC) constitue une des principales causes d'incapacité de l'adulte. Plus de $60 \%$ des personnes victimes d'un AVC perdront temporairement la capacité de marcher. Cet article offre un aperçu de l'évolution de la rééducation de la fonction locomotrice ainsi que de l'impact du développement des connaissances scientifiques et technologiques sur cette évolution. Ainsi, la pratique mentale, l'entraînement de la marche sur tapis roulant ainsi que l'immersion dans des environnements virtuels représentent des thérapeutiques fort prometteuses pour optimiser la récupération locomotrice et favoriser la réinsertion sociale post-AVC. <

\section{Nouvelles perspectives \\ en réadaptation motrice après un accident vasculaire cérébral}

Francine Malouin, Carol L. Richards, Bradford McFadyen, Julien Doyon

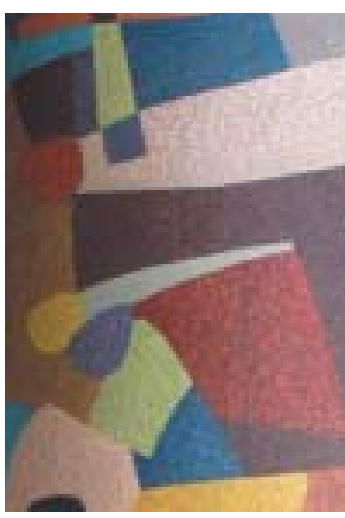

mer qu'un entraînement à la marche sur tapis roulant dès les premières semaines post-lésion est non seulement possible [3], mais souhaitable puisqu'il accélère la récupération locomotrice [4], ce qui a ouvert la voie à un entraînement précoce orienté vers la tâche. En effet, au cours des années 1990, les résultats d'une série d'études cliniques vérifiant divers modes d'entraînement combinant tapis roulant et support de poids ont confirmé la faisabilité d'un entraînement locomoteur précoce indépendamment de la sévérité de la déficience [5].

Des données expérimentales de plus en plus nombreuses justifient les bienfaits d'un entraînement orienté vers la tâche [6-8]. Cet entraînement préconise, outre la locomotion comme telle, le recours à des tâches qui s'apparentent à la marche et à des exercices constitués de composantes dérivées de la marche. Ainsi, monter et descendre d'un bloc, faire un pas vers l'avant, se dresser sur le bout des orteils constituent des exercices pour 
améliorer la force musculaire, la coordination, l'équilibre et la flexibilité, qui sont des qualités requises pour la marche [8]. S'exercer à marcher sur des surfaces diverses (tapis, gazon), négocier des rampes, monter et descendre des escaliers, parcourir des trajets parsemés d'objets ou peuplés de personnes y circulant font également partie de la rééducation locomotrice. Non seulement de telles pratiques augmentent la force musculaire et l'endurance, mais elles favorisent aussi l'adaptation aux exigences environnementales du milieu de vie du patient $[6,9,10]$. Plus récemment, l'attention s'est portée sur les mauvaises performances post-AVC concernant l'endurance à la marche. $\varepsilon$ n effet, la distance moyenne parcourue en six minutes par des personnes ayant subi un AVC correspondait à moins de
$50 \%$ de celle effectuée par des sujets témoins $[7,9]$. Le fait que cette diminution de l'endurance soit observée aussi chez des patients marchant à une vitesse normale révèle une carence particulière nécessitant un entraînement en vue d'améliorer l'endurance comme telle $[9,11]$.

Outre le type d'activités, l'intensité de l'entraînement (fréquence et durée des sessions) constitue également un facteur pouvant influencer les mécanismes de plasticité liés à la récupération motrice consécutive à une lésion cérébrale. En effet, à l'aide de techniques permettant de cartographier les aires corticales, il a été démontré, par microstimulation chez l'animal [12] et par stimulation magnétique transcrânienne chez l'humain [13], que le nombre de répétitions d'une action motrice avait une influence directe sur la réorganisation du cortex moteur contrôlant les muscles entraînés.

Puisque l'exécution fréquente d'une action demeure le meilleur moyen d'acquérir une habileté motrice ou d'améliorer sa performance, des séances d'entraînement supervisées, en groupe ou individuelles, devraient être accessibles. $0 r$, en pratique, on se rend compte que les patients en réadaptation active connaissent de longues périodes d'oisiveté et de solitude [8]. Toutefois, pratiquer davantage n'est pas toujours possible soit du fait de faiblesses musculaires localisées voire d'une faiblesse généralisée, soit à cause d'un manque de disponibilité des thérapeutes. Quoi qu'il en soit, les études suggèrent que la récupération motrice serait meilleure si les patients s'exerçaient davantage. II convient donc d'explorer des stratégies alternatives d'entraînement appliquées à tous les stades de la récupération motrice pour favoriser la répétition des mouvements tout en exigeant moins d'effort physique.

\section{Favoriser la répétition, peu importe le stade d'entraînement par la «pratique» mentale}

Figure 1. Exemple d'un test de chronométrie mentale illustrant la concordance (coefficient de corrélation de Pearson) entre la durée du mouvement imaginé et exécuté au cours d'une activité qui consiste à poser le pied sur un bloc de $7,5 \mathrm{~cm}$ de haut et à le replacer au sol. Chaque point représente la moyenne individuelle de trois séries de cinq répétitions chacune, pour les mouvements exécutés, soit avec le membre gauche ou droit (sujets témoins) et le membre parétique ou non-parétique (patients post-AVC). Bien que la corrélation soit plus forte chez les sujets témoins, elle était statistiquement significative $(p=0,0001)$ dans les deux groupes, suggérant que les sujets post-AVC démontrent également une bonne capacité d'imagerie motrice. Noter que le niveau de concordance est symétrique pour chacun des groupes. La ligne diagonale représente la relation hypothétique $1: 1$ des mesures.
La pratique mentale, qui consiste à répéter des mouvements imaginés, est utilisée depuis longtemps par les sportifs pour améliorer ou maintenir leur niveau de performance motrice [14]. L'imagerie motrice agirait sur la planification et la préparation du mouvement. Ainsi, la similarité de l'activité cérébrale lors du mouvement imaginé et exécuté [15], les 
modifications de l'activité cérébrale [16], ainsi que les changements de la cartographie cérébrale corticale [13] observés après la pratique mentale suggèrent qu'elle agit sur l'organisation cérébrale. Comme la pratique mentale produit des effets qui ne se révèlent qu'avec un peu de pratique physique [13], les meilleurs résultats ont été obtenus en combinant pratique physique et pratique mentale [14]. Avant d'utiliser la pratique mentale, il convient de s'assurer que le sujet est capable de se représenter mentalement une action (imagerie motrice). Comme la durée du mouvement imaginé est similaire à celle du mouvement exécuté [14, 15], les chercheurs se sont appuyés sur la chronométrie mentale pour estimer la capacité d'imagerie motrice (Figure 1). A l'exception de certains cas de lésion du cortex pariétal [17], les sujets porteurs d'une lésion cérébrale présentent [18] une assez bonne concordance temporelle entre le geste imaginé et le geste exécuté (Figure 1), suggérant qu'ils pourraient bénéficier de pratique mentale. Effectivement, on a rapporté une amélioration de la fonction du membre supérieur [19] et de l'utilisation de la jambe affectée lors du leverdebout [20] grâce à la pratique mentale post-AVC. Aucune étude à ce jour n'a vérifié les effets de la pratique mentale sur la locomotion comme telle. Toutefois, le fait que l'imagination de tâches locomotrices (Figure 2), comme amorcer la marche, marcher et marcher en contournant des obstacles, active un réseau de structures cérébrales communes aux activités locomotrices [21], suggère que la pratique mentale puisse être applicable à l'entraînement locomoteur. Celle-ci présente de multiples avantages du fait que le sujet peut s'y adonner fréquemment et à son gré, à tous les stades de la rééducation fonctionnelle, et ce, sans les contraintes de la pratique physique. Cependant, la pratique mentale ne remplace pas la pratique physique et n'est pas applicable chez tous les sujets $[17,20]$.

\section{Préparer aux contraintes environnementales de la vie quotidienne par le développement de scénarios et de stratégies d'entrainement en réalité virtuelles}

La rééducation de la fonction locomotrice embrasse un ensemble de conditions préparant le patient à s'adapter aux contraintes environnementales auxquelles il sera confronté à son retour dans la société. On doit exposer le patient dès que possible à des situations l'obligeant à sélectionner des stratégies appropriées pour circuler en toute sécurité dans des environnements changeants et parsemés d'obstacles physiques et de distractions sensorielles. L'utilisation de situations combinant des éléments d'incertitude et de surcharge attentionnelle doit aussi également être considérée puisque celles-ci modifient les stratégies anticipatoires d'évitement d'obstacles (Figures 3A, $3 B$ ). De telles informations sur le comportement locomoteur anticipatoire sont nécessaires pour le développement de scénarios et d'environnements destinés à l'entraînement locomoteur de stratégies anticipatoires d'évitement. Malheureusement, compte tenu des exigences qu'une telle diversité d'environnements commande, l'entraînement de ces stratégies anticipatoires est difficilement réalisable en clinique. Cependant, l'avènement de la réalité virtuelle combinant tapis roulant, support de poids et immersion dans un environnement virtuel permettrait, même aux patients sévèrement atteints, d'accéder à un tel entraînement (Figure 3C). Bien que l'utilisation d'environnements virtuels en réadaptation soit récente, les résultats suggèrent qu'il y a bien transfert des apprentissages aux situations réelles tant chez l'enfant [23] que chez l'adulte [24, 25].

\section{Perspectives et conclusions}

La pratique mentale et la réalité virtuelle offrent des voies fort prometteuses pour optimiser la récupération locomotrice et favoriser la réinsertion sociale des

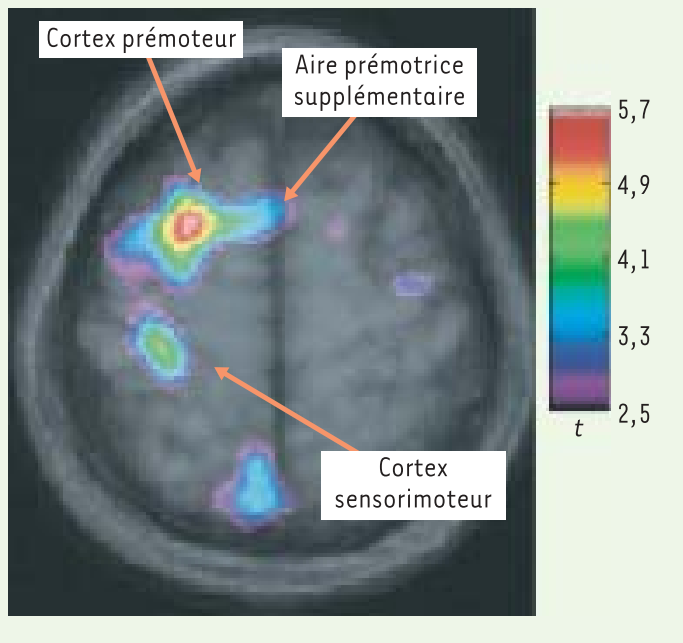

Figure 2. Représentation combinée des images de tomographie par émission de positons et d'imagerie par résonance magnétique illustrant l'augmentation du débit sanguin lors de la marche imaginée. Cette section représente la moyenne de la condition imaginée (marche) moins la condition témoin (repos) de six sujets. L'amplitude des activations focales est indiquée par le code des couleurs. 
patients victimes d'AVC. L'évaluation de leurs applications respectives en clinique aidera à mieux cerner leurs avantages, leurs limites et leur complémentarité. Cependant, si l'une est peu coûteuse et d'application facile, l'autre repose sur l'accessibilité à une technologie avancée et sur l'aide d'un personnel hautement qualifié. $\diamond$

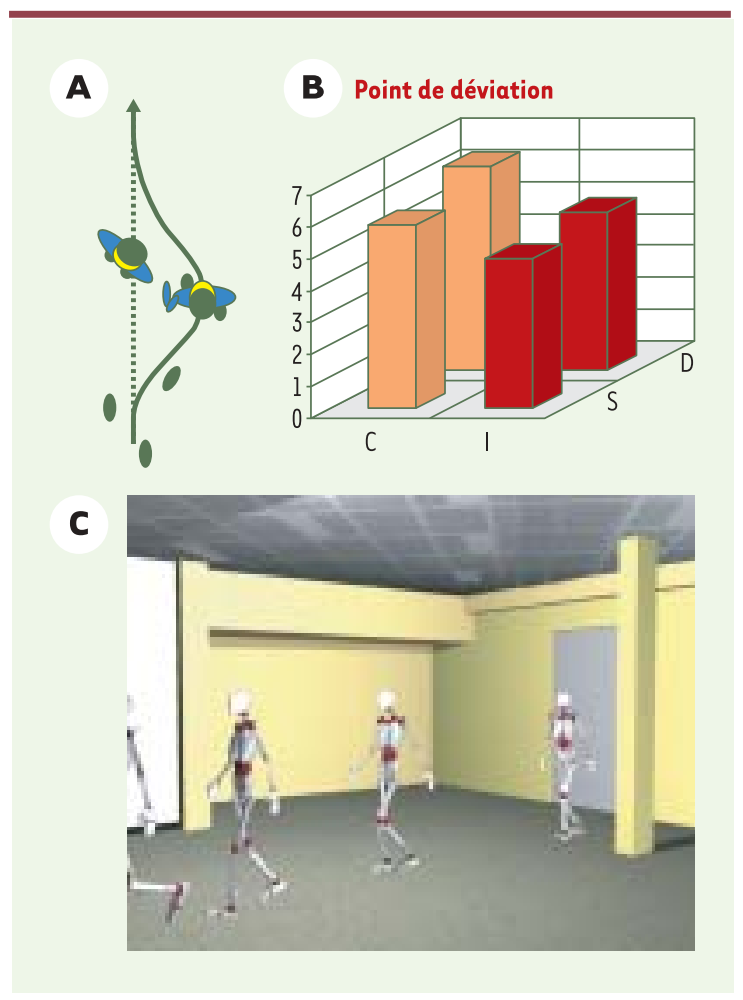

Figure 3. A. Schéma illustrant la déviation de la trajectoire de marche (stratégie d'évitement) lorsque le sujet contourne un obstacle de forme humaine. B. Nombre de pas (moyenne de cinq sujets) entre le point où la déviation du sujet débute et la position de l'obstacle lorsque la présence de l'obstacle est connue (C) ou inconnue (I), et lorsque le sujet est distrait (D) ou non distrait (S) par un message verbal. Lorsque le sujet est informé de la présence de l'obstacle (C), le nombre de pas est plus grand que lorsqu'il l'ignore (I) indiquant qu'il amorce sa stratégie d'évitement plus tôt (anticipation) lorsqu'il est informé. L'ajout d'un message verbal amène le sujet à anticiper davantage sa stratégie d'évitement (nombre de pas avant I'obstacle augmente), suggérant que la distraction incite à plus de prudence. $C$. Représentation schématique d'un environnement virtuel programmé mettant en scène des personnes entravant le trajet du sujet; cet environnement est visualisé en trois dimensions par l'intermédiaire d'un casque de réalité virtuelle.

\section{REMERCIEMENTS}

Les auteurs désirent remercier les organismes de subvention suivants : le FRSQ, Santé Canada et le CRSNG, les patients, ainsi que Francine Dumas, thérapeute de recherche, Daniel Tardif, assistant de recherche et Martin Gérin-Lajoie, étudiant au doctorat.

\section{SUMMARY}

To walk again after a stroke: new perspectives of locomotor rehabilitation

The task-oriented approach incorporating treadmill walking for retraining gait early after stroke has contributed to promote locomotor recovery. To augment practice, training strategies such as mental practice and training in virtual environments are proposed. While the former offers more practice with less physical exertion, the latter allows safe practice in a variety of challenging environments. Work is under way to assess whether these new strategies can further enhance locomotor recovery. $\diamond$

\section{RÉFÉRENCES}

1. Jorgensen HS, Nakayama $H$, Raaschou HO, Olsen TS. Recovery of walking function in stroke patients. The Copenhagen Stroke Study. Arch Phys Med Rehabil 1995 ; 76 : 27-32.

2. Desrosiers J, Noreau L, Rochette A, Bravo G, Boutin C. Predictors of handicap situations following post-stroke rehabilitation. Disabil Rehabil 2002 ; 24 : 774-85.

3. Malouin F, Potvin M, Prevost J, Richards CL, Wood-Dauphinee $S$. Use of an intensive task-oriented gait training program in a series of patients with acute cerebrovascular accidents. Phys Ther 1992 ; 72 : 781-9.
4. Richards CL, Malouin F, Wood-Dauphinee $S$, Williams JI, Bouchard JP, Brunet D. Task-specific physical therapy for optimization of gait recovery in acute stroke patients. Arch Phys Med Rehabil 1993 ; 74 612-20.

5. Visintin M, Barbeau $H$, Korner-Bitensky N. A new approach to retrain gait in stroke patients through body weight support and treadmill stimulation. Stroke 1998 ; 29 :

1122-8.

6. Dean CM, Richards $C L$ Malouin F. Task-related circuit training improves performance of locomotor tasks in chronic stroke. A randomized controlled pilot trial. Arch Phys Med Rehabil 2000 ; 81 : 409-17. 
7. Richards CL, Malouin F, Dean C. Gait in stroke: assessment and rehabilitation. In: Duncan $\mathrm{P}$, ed. Clinics in geriatric medicine.

Philadelphie:

W.B. Saunders, 1999 : $15: 833-55$

8. Carr JH, Shepherd RB. Neurological rehabilitation. Optimizing motor performance. 0xford: Butterworth Heinemann, 1998.

9. Dean C, Richards CL, Malouin F. Walking speed over 10 meters overestimates locomotor capacity after stroke. Clin Rehabil 2001 ; 15 : 415-21.

10. Smith GV, Silver KH, Goldberg AP, Macko RF. «Task-oriented» exercise improves hamstring strength and spastic reflexes in chronic stroke patients. Stroke 1999 ; 30 : 212-9.

11. Macko RF, Smith GV, Dobrovolny CL, Sorkin JD, Goldberg AP, Silver KH. Treadmill training improves fitness reserve in chronic stroke patients. Arch Phys Med Rehabil 2001 ; 82 : 879-84.

12. Nudo RJ, Pautz \&J, Frost SB. Role of adaptive plasticity in recovery of function after damage to motor cortex. Muscle Nerve 2001 ; 24 : 1000-19.

13. Pascual-Leone A, Nguyet D, Cohen LG, Brasil-Neto JP, Cammarota A, Hallett $M$. Modulation of muscle responses evoked by transcranial magnetic stimulation during the acquisition of new fine motor skills. J Neurophysiol 1995 ; 74 : 1037-45.
14. Jackson PL, Lafleur M, Malouin F, Richards CL, Doyon J. Potential role of mental practice using motor imagery in neurologic rehabilitation. Arch Phys Med Rehabil 2001 ; 82 : 1133-41.

15. Jeannerod, $M$. The representing brain: Neural correlates of motor intention and imagery. Behav Brain Sci 1994 ; 17 : 187-245.

16. Jackson PL, Lafleur MF, Malouin F, Richards CL, Doyon J. Functional cerebral reorganization following motor sequence learning through mental practice with motor imagery. Neurolmage 2003 (sous presse).

17. Sirigu A, Duhamel JR, Cohen L, Pillon B, Dubois B, Agid $Y$. The mental representation of hand movements after parietal cortex damage. Science 1996 ; 273 : 1564-8.

18. Malouin F, Desrosiers J, Doyon J, Richards CL. Motor imagery ability after stroke. Soc Neurosci 2001 ; 27 : 950.4 (abstract).

19. Page SJ, Levine P, Sisto SA, Johnston MV. Mental practice combined with physical practice for upperlimb motor deficit in subacute stroke. Phys Ther $2001 ; 81$ : 1455-62.
20. Malouin F, Belleville $S$, Descrosiers J, Doyon J, Richards CL. Working memory and mental practice after stroke. Arch Phys Med Rehabil 2003 (sous presse).

21. Malouin F, Richards CL, Jackson PL, Dumas F, Doyon J. Brain activations during motor imagery of locomotor-related tasks: a PET study. Hum Brain Mapp 2003 ; 19: 47-62.

22. Gérin-Lajoie M, Richards $\mathrm{CL}$, McFadyen BJ. Walking around upright obstacles: Effects of obstacle mobility and division of attention on anticipatory locomotor strategies. Soc Neurosci 2001 ; $27: 406.124$ (abstract).

23. McComas J, Pivik J. Laflamme M. Current uses of virtual reality for children with disabilities. Stud Health Technol Inform 1998 ; 58 :161-9.
24. Holden M, Dyar T, Callahan J, Schwamm L, Bizzi $\varepsilon$. Quantitative assessment of motor generalization in the real world following training in virtual environment. Neurol Rep 2001 ; 25 : 130-1.

25. DeRugy A, Montagne G, Buekers MJ, Laurent M. The study of locomotor pointing in virtual reality: The validation of a test set-up. Behav Res Meth Instr Comput 2000 ; 32 : 515-20.
TIRÉS À PART

F. Malouin 